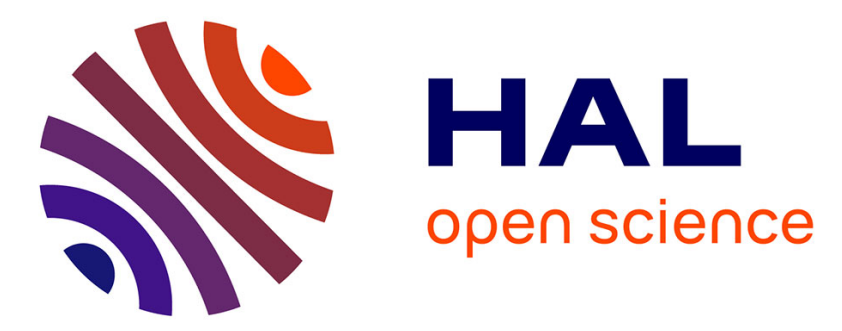

\title{
Vortex terahertz wave generation in air by femtosecond optical vortex pulses
}

\author{
Maksym Ivanov, Illia Thiele, Luc Bergé, Stefan Skupin, Danas Buožius, \\ Virgilijus Vaičaitis, Virgilijus Vaicaitis
}

\section{- To cite this version:}

Maksym Ivanov, Illia Thiele, Luc Bergé, Stefan Skupin, Danas Buožius, et al.. Vortex terahertz wave generation in air by femtosecond optical vortex pulses. Terahertz Emitters, Receivers, and Applications X, Aug 2019, San Diego, United States. pp.9, 10.1117/12.2529247 . hal-02282663

\section{HAL Id: hal-02282663 https://hal.science/hal-02282663}

Submitted on 10 Sep 2019

HAL is a multi-disciplinary open access archive for the deposit and dissemination of scientific research documents, whether they are published or not. The documents may come from teaching and research institutions in France or abroad, or from public or private research centers.
L'archive ouverte pluridisciplinaire HAL, est destinée au dépôt et à la diffusion de documents scientifiques de niveau recherche, publiés ou non, émanant des établissements d'enseignement et de recherche français ou étrangers, des laboratoires publics ou privés. 


\title{
Vortex terahertz wave generation in air by femtosecond optical vortex pulses
}

\author{
Maksym Ivanov $^{\mathrm{a}}$, Illia Thiele ${ }^{\mathrm{b}}$, Luc Bergéc ${ }^{\mathrm{c}}$, Stefan Skupin ${ }^{\mathrm{d}}$, Danas Buožius ${ }^{\mathrm{a}}$, and Virgilijus \\ Vaičaitis $^{\text {a }}$ \\ ${ }^{a}$ Laser Research Center, Vilnius University, 10 Sauletekio avenue, Vilnius, Lithuania, LT-10223 \\ b Department of Physics, Chalmers University of Technology, Göteborg, Sweden, SE-412 96 \\ ${ }^{\mathrm{c}} \mathrm{CEA}, \mathrm{DAM}$, Arpajon, France, DIF - 91297 \\ ${ }^{\mathrm{d}}$ Institut Lumiere Matiere, UMR 5306 Universit Lyon 1 - CNRS, Universit de Lyon, \\ Villeurbanne, France, F-69622
}

\begin{abstract}
This proceeding details the experimental investigation and scheme used to generate vortex beams at $\mathrm{THz}$ and far-infrared frequencies. Main experimental and numrical results are presented by M. Ivanov et al. ${ }^{1,2}$ We investigate the generation of broadband terahertz $(\mathrm{THz})$ pulses between 10 and $40 \mathrm{THz}$ with a phase singularity from two-color femtosecond laser-induced gas-plasmas. Experimental scheme allows for independent control of the amplitude, phase, polarization, and longitudinal and transverse positions of fundamental and second harmonics. When the second harmonic beam has a vortex charge the $\mathrm{THz}$ beam acquires an azimuthal phase modulation as well. Moreover, it turns out that also the THz intensity becomes modulated along the azimuthal angle. The phase structure of the singular $\mathrm{THz}$ beam is indirectly revealed by astigmatic transformation of the beam by a cylindrical mirror and additionally confirmed by the vortex $\mathrm{THz}$ beam behaviour upon change of relative phase between the fundamental and the second harmonic.
\end{abstract}

Keywords: Terahertz radiation, Optical Vortex, Plasma, THz, phase singularity

\section{INTRODUCTION}

Terahertz $(\mathrm{THz})$ radiation is of great current interest due to many applications such as nonlinear THz spectroscopy and imaging ${ }^{3}$ or electron bunch compression. ${ }^{4}$ One of the compact and effective methods to obtain very high THz field strengths and extremely broadband spectral widths is $\mathrm{THz}$ wave generation from plasma filaments formed in air by focused bichromatic femtosecond laser pulses consisting of first and second harmonic waves $(\mathrm{FH}, \mathrm{SH}) \cdot{ }^{5-9}$ On the other hand, special light fields, such as optical vortex ${ }^{10}$ radially polarized,${ }^{11}$ Bessel ${ }^{12,13}$ and Airy beams ${ }^{14}$ are widely studied and employed in various fields. ${ }^{15,16}$ Previous attempts on vortex generation at the $\mathrm{THz}$ frequencies ${ }^{17-24}$ were exclusively based on manipulation of $\mathrm{THz}$ wave by external components which are inherently limited in terms of acceptable bandwidth. Therefore, alternative methods for ultra-broadband vortex generation at $\mathrm{THz}$ frequencies should be proposed and investigated. Very recently, vortex $\mathrm{THz}$ have been generated without external shaping elements ${ }^{25,26}$ in ZnTe crystal, with comparably narrowband spectrum. Therefore, in this work we investigated a novel method of vortex $\mathrm{THz}$ pulse generation, already at the $\mathrm{THz}$ generation stage, emitted from the air-plasma induced by FH and SH. SH carrying an optical vortex charge affects not only phase but also intensity distribution of the generated $\mathrm{THz}$ pulse. Results of our investigation suggest an alternative method for the generation of vortex $\mathrm{THz}$ wave spanning ultra-broad band frequency range, which is not limited by the spectral acceptance of the external shaping elements or down-converting crystals. This method can be used for active control of the $\mathrm{THz}$ pulse properties, giving great flexibility for applications of $\mathrm{THz}$ radiation. Main experimental results with comprehensive numerical simulations are presented by M. Ivanov et al., ${ }^{1,2}$ in this proceeding we present details of the experimental investigation and related scheme. 


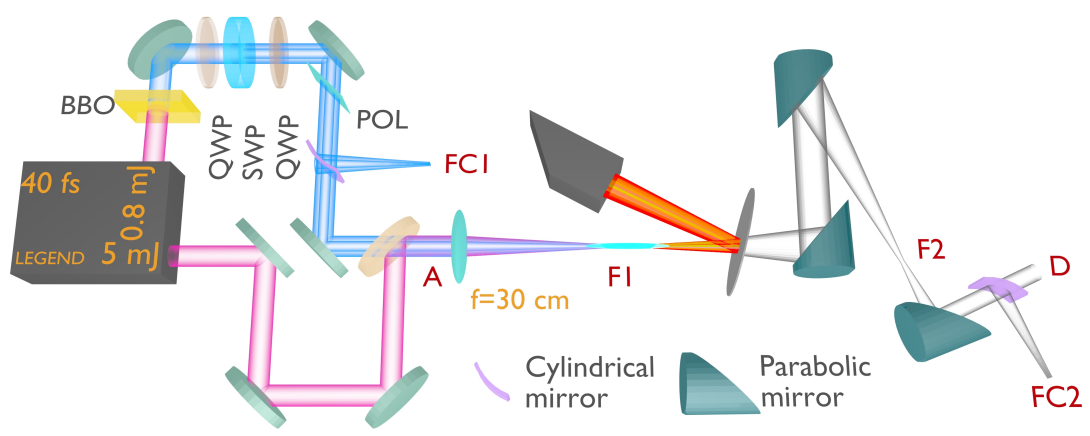

Figure 1. Sketch of experimental setup. QWP - quarter wave plate, SWP - S-waveplate (q-plate), POL - polarizer, BBO - nonlinear crystal for SH generation. Parabolic and cylindrical mirror are labled in the Figure. SH is shown by blue, FH by red-pink, THz radiation - by gray color. Red letters label positions at which experimental images are taken.

\section{EXPERIMENTAL OBSERVATIONS}

The experimental setup in sketched in Fig. 1. We have used a $1 \mathrm{kHz}$ repetition rate laser system (Legend elite duo $\mathrm{HE}+$, Coherent Inc.). Right before main focusing lens (silica, $f=30 \mathrm{~cm}$ ) labeled 'A' in the setup, $\mathrm{FH}$ had central wavelength of $790 \mathrm{~nm}$, FWHM pulse duration $50 \mathrm{fs}$ and energy of $6 \mathrm{~mJ}$. In this position SH had central wavelength of $395 \mathrm{~nm}$, FWHM pulse duration $\sim 50 \mathrm{fs}$ and energy of $60 \mu \mathrm{J}$ in Gaussian state and $50 \mu \mathrm{J}$ in vortex state. Gaussian SH was converted to an optical vortex by a method described in, ${ }^{27}$ which ensures more than octave spectral bandwidth of vortex generation. First SH was circularly polarized by a quarter wave plate (QWP) and converted to an optical vortex (OV) beam by an S-waveplate (RPC-405-06-557, Workshop of Photonics) (SWP). Subsequent polarization filtering by a second quarter wave plate (QWP) and a polarizer (POL) ensured generation of a linearly polarized vortex in femtosecond SH beam over all spectral bandwidth of the SH pulse. A $0.5 \mathrm{~mm}$ thick Si wafer along with various commercial $\mathrm{THz}$ filters was used to remove the high frequency part and to transmit only $\mathrm{THz}$ radiation, which was collimated and shrank by parabolic mirrors in a telescope configuration to match the detector aperture. Imaging of the generated $\mathrm{THz}$ beam was performed with a thermal camera detector (VarioCAM head HiRes 640, InfraTec GmbH), sensitive in the range $0.140 \mathrm{THz}$ (3000 7.5 $\mu \mathrm{m}$ ). Spectra of $\mathrm{THz}$ radiation were obtained from Fourier transformed interferometric measurements of $\mathrm{THz}$ radiation using pyroelectric detector (TPR-A-65 THz, Spectrum Detector Inc.), sensitive in the range 0.1 - $300 \mathrm{THz}(3000-1 \mu \mathrm{m})$ with a flat response function from $\sim 3$ to $\sim 100 \mathrm{THz}$. Typically generated $\mathrm{THz}$ radiation spectrum spans from 10 to $40 \mathrm{THz}$ (right-bottom panel of Fig. 9). Deeps in the spectrum are associated with absorbance in the guiding elements such as Si filters and pellicle beam splitters. The efficiency of $\mathrm{THz}$ generation with the regular Gaussian pulses was about $10^{-4}$, but dropped to about $10^{-5}$ in the case of the SH vortex pump due to the different spatial intensity distributions of the Gaussian FH and vortex SH beams.

Since our experimental scheme allows for independent control of the amplitude, phase, polarization, and longitudinal and transverse positions of $\mathrm{FH}$ and $\mathrm{SH}$ then before commencing experiments on the generation of vortex $\mathrm{THz}$, we test performance of the setup versus misalignment of the above mentioned parameters. Therefore, spectrum of the generated $\mathrm{THz}$ radiation was tested for lateral, transverse, and temporal misalignment between $\mathrm{FH}$ and $\mathrm{SH}$, and also versus energy of $\mathrm{FH}$. Results suggests that change of power of $\mathrm{FH}$, lateral and transverse misalignment between $\mathrm{FH}$ and $\mathrm{SH}$ does not affect significantly the spectral distribution of the generated $\mathrm{THz}$ beam, in contrast to the change of delay between FH and SH pulses, which shift frequency peak of the generated $\mathrm{THz}$ radiation.

Further author information: (Send correspondence to M. I.)

M.I.: E-mail: maks.ivannov@gmail.com, Telephone: N/A 


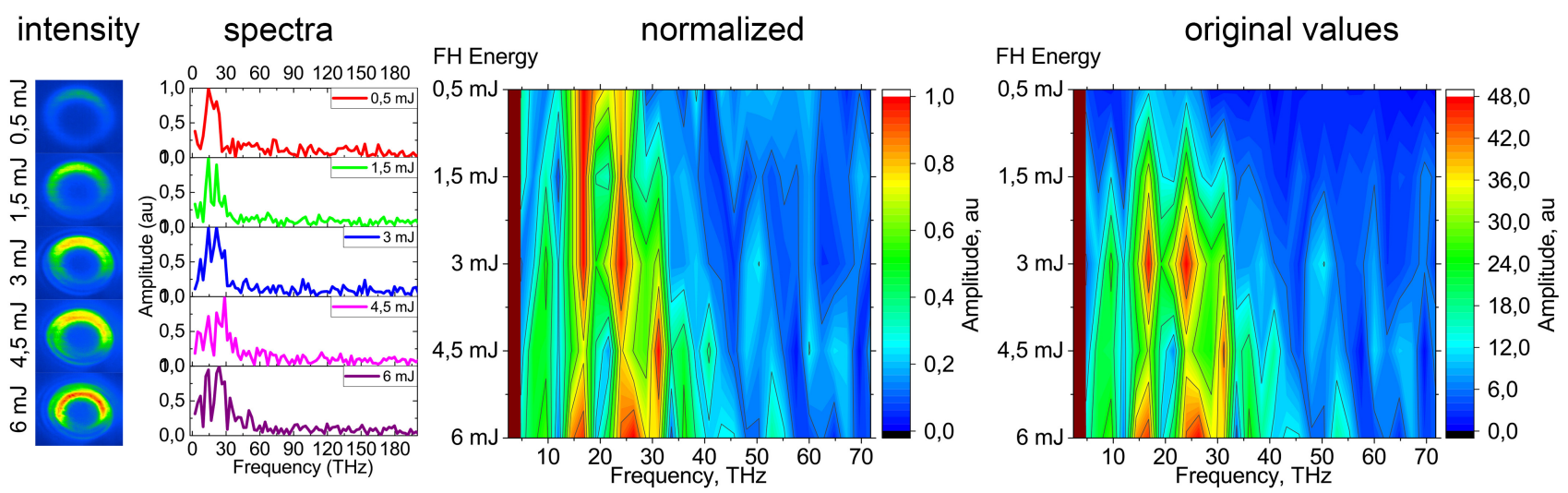

Figure 2. Experimental images and spectra of the generated $\mathrm{THz}$ beam versus energy of FH. Energy of SH is constant $60 \mu \mathrm{J}$, time delay is $0 \mathrm{ps}$. See text for details.

Spectra of THz beam versus energy of FH is shown in Fig. 2. Energy of SH kept constant at $60 \mu \mathrm{J}$. Column 'spectra' shows spectra of the generated THz beam at the specified energy levels of FH. Insets to the left from this column show intensity distribution of the generated $\mathrm{THz}$ beam at specified energy levels. Two rightmost columns additionally show spectral maps with original (non-normalized) scale ('original values') and normalized data ('normalized'). These maps are automatically generated in an OriginLab software using data from the column 'spectra'. The data points between these six experimentally measured data sets (those at $6,4.5,3,1.5$, and $0.5 \mathrm{~mJ}$ ) are created automatically by the OriginLab software for smooth transition between these data sets. As is visible from from Fig. 2, increasing the energy of $\mathrm{FH}$ from $0.5 \mathrm{~mJ}$ to $6 \mathrm{~mJ}$ broadens the spectrum of the generated $\mathrm{THz}$ wave from $13.5 \mathrm{THz}$ to $28.5 \mathrm{THz}$ at FWHM, correspondingly, and produces higher $\mathrm{THz}$ yield. At any incident energy level of $\mathrm{FH}$ the spectrum of the generated $\mathrm{THz}$ wave peaks at 13 and $25 \mathrm{THz}$ (Fig. 2). Additionally, from the image insets of the $\mathrm{THz}$ intensity distributions, one can notice slight decrease in the radius of the generated $\mathrm{THz}$ radiation with increase of the energy of $\mathrm{FH}$.

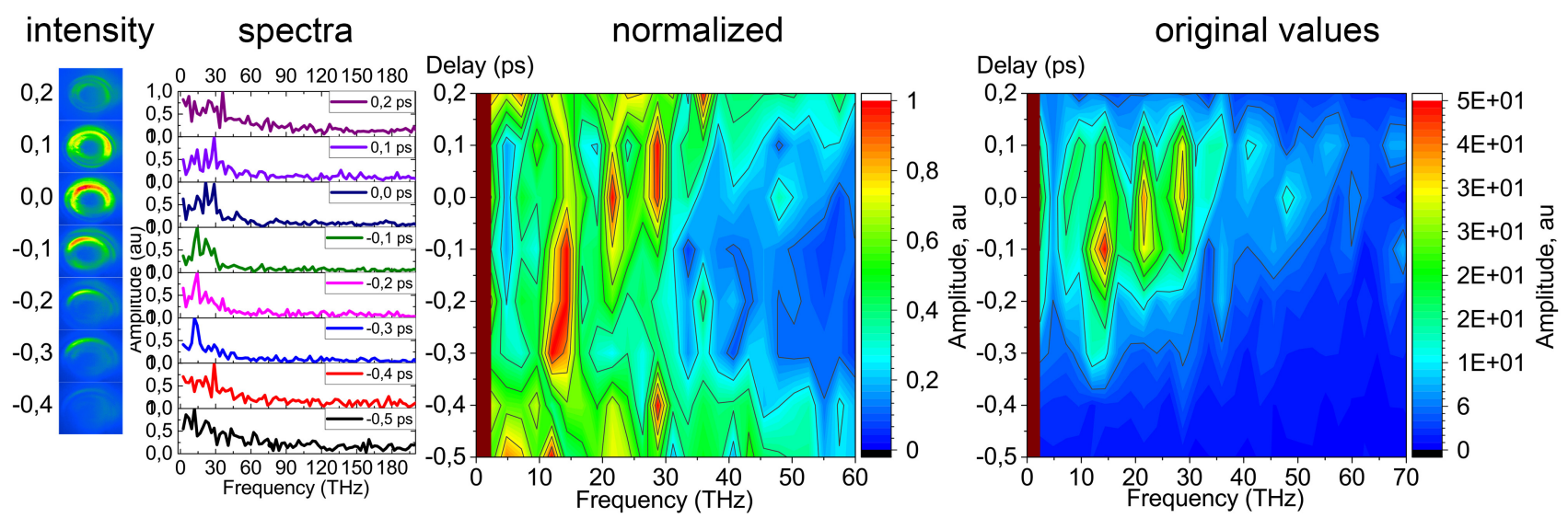

Figure 3. Experimental images and spectra of $\mathrm{THz}$ beam versus time delay between $\mathrm{FH}$ and SH. Energy of $\mathrm{FH}$ is $6 \mathrm{~mJ}$, $\mathrm{SH}$ is $60 \mu \mathrm{J}$. See text for details.

Effect of the temporal delay of time of arrival of $\mathrm{FH}$ with respect to that of $\mathrm{SH}$ pulse on spectra of the generated $\mathrm{THz}$ beam is shown in Fig. 3. Time of arrival of $\mathrm{FH}$ was changed from simultaneous arrival $(0 \mathrm{ps})$ to $0,2 \mathrm{ps}$ in positive direction, meaning $\mathrm{FH}$ arrives prior to $\mathrm{SH}$, and to $-0.5 \mathrm{ps}$ in the negative direction, meaning $\mathrm{FH}$ arrives after SH pulse to the interaction point. As seen from the normalized and non-normalized frequency maps (on the right in Fig. 3) the maximum of the THz yield is at 0 and -0.1 ps delay. As delay changes from 0.2 ps to $-0.5 \mathrm{ps}$ ( $\mathrm{FH}$ arrives prior to and after $\mathrm{SH}$, correspondingly) maximum peak of the generated $\mathrm{THz}$ radiation shifts from higher to lower $\mathrm{THz}$ frequencies, namely from $36 \mathrm{THz}$ at $0.2 \mathrm{ps}, 21.5 \mathrm{THz}$ at $0 \mathrm{ps}$, to $12 \mathrm{THz}$ at 
-0.5 ps. This dependence is attributed to the absorption and reflection of the lower frequencies by the plasma filament developed in the focal area from the focused FH pulse. Earlier arrival of FH harmonic leads to more dense/developed plasma, therefore more low frequency components are absorbed/reflected. One can notice that spectrum at -0.4 ps does not follow above described trend of the gradual frequency shift. We believe that this is due to the experimental error rather than to some physical effect.
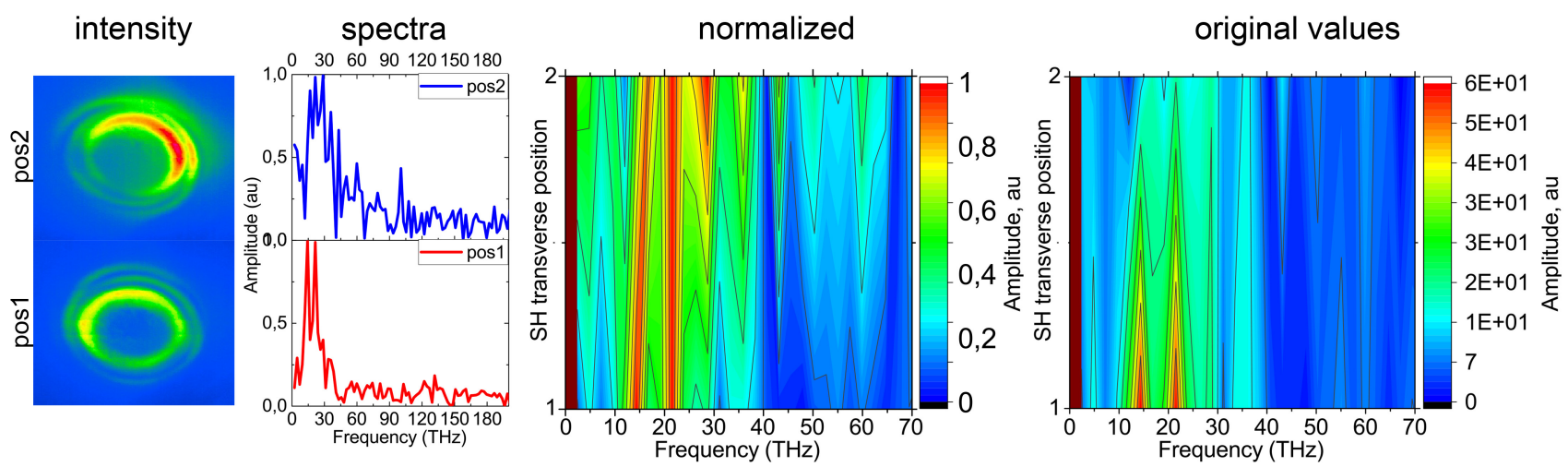

Figure 4. Experimental images and spectra of $\mathrm{THz}$ beam versus transverse alignment of $\mathrm{FH}$ and $\mathrm{SH}$ beams. Energy of FH is $6 \mathrm{~mJ}$, SH is $60 \mu \mathrm{J}$, time delay is 0 ps. See text for details.

Misalignment of superposition of $\mathrm{FH}$ and $\mathrm{SH}$ beams in the focal spot in the transverse direction is shown in Fig. 4. Transverse misalignment of FH and $\mathrm{SH}$ affects symmetry of conically emitted ring shaped $\mathrm{THz}$ intensity distribution. Image inset at the bottom ('pos1') correspond to a good alignment, symmetric intensity distribution of the generated $\mathrm{THz}$ beam, and inset at the top show asymmetric $\mathrm{THz}$ beam corresponding to poor transverse alignment ('pos2'). Symmetric THz radiation has higher $\mathrm{THz}$ yield as seen from non-normalized frequency map ('original values'). Spectrum of symmetric $\mathrm{THz}$ beam has maxima at $14.5 \mathrm{THz}$ and $21.5 \mathrm{THz}$, while spectrum of asymmetric $\mathrm{THz}$ beam is slightly shifted towards the higher frequencies and peaks at $21.5 \mathrm{THz}$ and $29 \mathrm{THz}$.

To test the properties of the generated $\mathrm{THz}$ beam versus spatial alignment of the $\mathrm{FH}$ and $\mathrm{SH}$ pulses we have slightly modified the experimental setup. We have introduced a telescope to the path of SH to control its longitudinal position in the focus of the main lens F1 (1) by changing the spatial size of the SH beam passing through the main focusing lens. Dispersion of lenses in the telescope has prolonged duration of the SH pulse to $\approx 150 \mathrm{fs}$. Spatial focusing along propagation direction is shown inthe upper part of Fig. 5 . When taking these images energies of harmonics were reduced to the level lower than the damage threshold of the CCD camera beam profiler. As seen from this figure upon focusing $\mathrm{FH}$ and $\mathrm{SH}$ beams have different divergence angles.

Generation of $\mathrm{THz}$ radiation versus the misalignment of superposition of $\mathrm{FH}$ and $\mathrm{SH}$ beams in the focal spot in the lateral direction was tested by moving the SH focal spot with respect to that of FH over the distance of 4.5 $\mathrm{mm}$, with $0.5 \mathrm{~mm}$ step. Results are shown in Fig. 5. Image of FH labelled with 0 correspond to focal position of $\mathrm{FH}$. After focusing both $\mathrm{FH}$ and $\mathrm{SH}$ develops as a conical emission with generation of a supercontinuum (images labelled 1 and 2 in the upper part of the figure). The upper part of this figure demonstrates two case of extreme alignment. First case, when focused FH overlaps with already expanding in a conical shape SH generating supercontinuum (shown on the left, $\mathrm{FH}$ '0' correspond to SH '2'). Second case, when focused FH overlaps with not fully focused yet SH beam (shown on the right, $\mathrm{FH}$ '0' correspond to SH '-2'). Lower part of Fig. 5 shows intensity and spectra of generated $\mathrm{THz}$ beam. Labelling marks position of $\mathrm{SH}$ with respect of '0' position of FH. Spectrum labelled '0' corresponds to the optimal alignment (harmonics overlap in the focus, ' 0 ' of FH harmonic overlaps with ' 0 ' of SH) and the highest THz yield, as clearly visible in the non-normalized rightmost spectral map. Images inserted on the left show that the intensity distribution of the generated $\mathrm{THz}$ beam does not change significantly with change of the lateral alignment. Measured spectra, as clearly visible from the normalized spectral map, suggests that peak of the generated $\mathrm{THz}$ beam does not shift significantly nor consistently, and peaks at approximately the same frequencies regardless of the longitudinal alignment.

During this experiment the FH beam was always kept Gaussian. Experimental images of the SH beam are shown in Fig. 6. When taking these images energies of harmonics were reduced to the level lower than the 

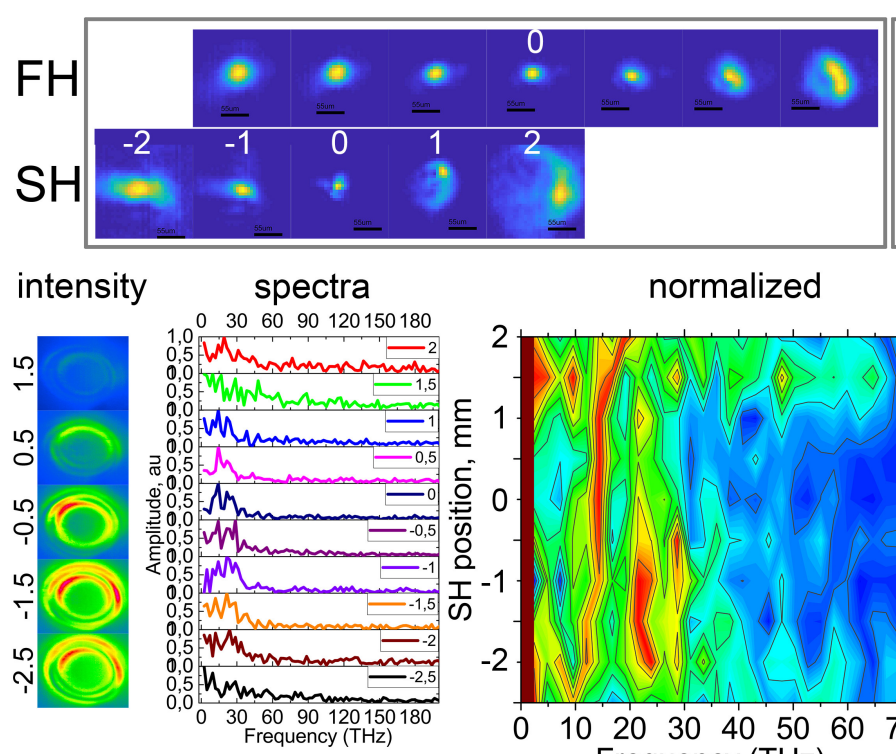

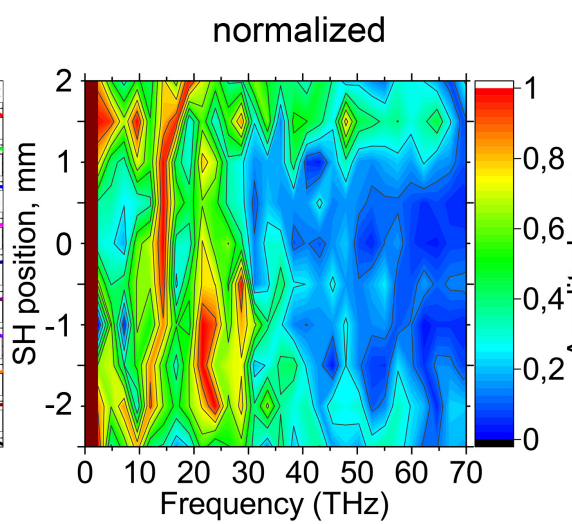

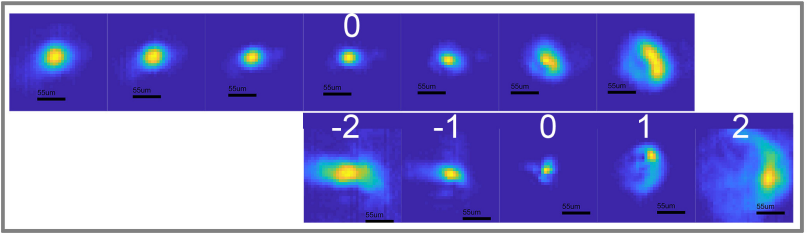

original values

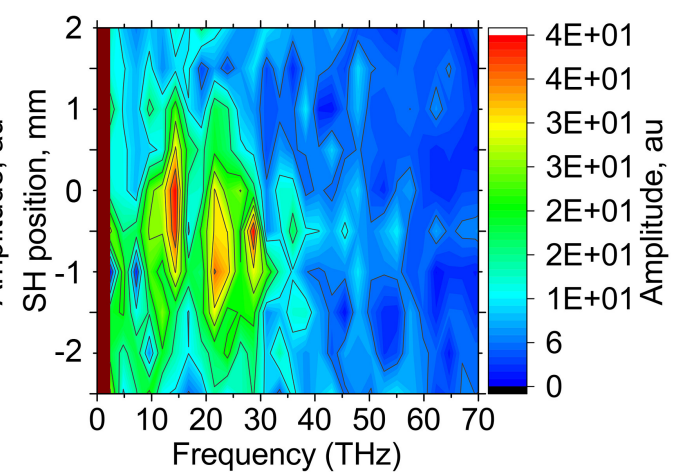

Figure 5. Upper part: Experimental images of the FH and $\mathrm{SH}$ upon focusing along propagation direction. Numbers mark positions of images with respect to the focus $(0 \mathrm{~mm})$ in millimeters. Lower part: Experimental images and spectra of $\mathrm{THz}$ beam versus lateral alignment of $\mathrm{FH}$ and $\mathrm{SH}$ focal spots. Energy of $\mathrm{FH}$ is $6 \mathrm{~mJ}$, SH is $60 \mu \mathrm{J}$, time delay is 0 ps. See text for details.

damage threshold of the CCD camera beam profiler. The first and the second row present the SH Gaussian ('SH') and vortex beam ('SHv'), respectively. The first column 'A' shows images of harmonics before the main focusing lens at position A (see Fig. 1); the second column 'F1' - in the focus of that lens (position F1 in Fig. 1). The FWHM beam widths are 33 and $38 \mu \mathrm{m}$ for the $\mathrm{FH}$ and the vortex $\mathrm{SH}$ in the focus of the main focusing lens, respectively. The third column 'FC1' in Fig. 6 shows images of the SH beam in the focus of a cylindrical mirror, $f=200 \mathrm{~mm}$ ('FC1' in Fig. 1). The top image in the 'FC1' column, presents a straight line profile as expected for a focused Gaussian SH beam; the bottom image shows an interrupted line corresponding to a SH vortex beam in the focus of the cylindrical mirror. The number of the dark stripes is equal to the modulus of the vortex topological charge, ${ }^{28}$ which in our case is $\left|l_{S H}\right|=1$. The method of vortex detection by astigmatic transformation of singular beams in the focus of cylindrical lens shows different intensity patterns for the regular and vortex beams. This method is well-developed ${ }^{28}$ and gives the same results (allows to measure topological charge) as another well-known interferometric vortex detection method, where forked fringes are observed in intensity patter of interference. Experimental images of the generated THz beams are shown in Fig. 7. The first row labeled ' $\mathrm{THz}$ ' shows intensity distributions of the conical $\mathrm{THz}$ beam obtained in the case of Gaussian $\mathrm{SH}$, similar to those reported previously. ${ }^{29,30}$ Intensity distributions of the $\mathrm{THz}$ beam in the second row labeled as 'THz vortex' were acquired when the SH was an OV. The column labeling corresponds to locations indicated in Fig. 1. Column 'F2' shows the intensity distribution of the $\mathrm{THz}$ beam in the focus of the second parabolic mirror. As expected, the $\mathrm{THz}$ beam without phase singularity has a maximum intensity in the center of the beam, while intensity is minimum in the focus in case of vortex $\mathrm{THz}$ beam. The column ' $\mathrm{D}$ ' shows the collimated $\mathrm{THz}$ beam. As expected, the $\mathrm{THz}$ beam without phase singularity has a symmetric ring intensity distribution, while the $\mathrm{THz}$ beam with phase singularity has an intensity modulation manifesting as two maxima along the azimuthal angle, in agreement with our theoretical predictions $\left({ }^{1,2}\right)$. The $\mathrm{THz}$ beam patterns obtained by the thermal camera in fact are averaged over the full $\mathrm{THz}$ spectral range obtained (0.1-40 THz). Column 'FC2' shows the THz intensity distribution in the focal plane of the cylindrical mirror $(f=200 \mathrm{~mm})$. The single bright line in the Fig. 7 'FC2' first row suggests that the generated $\mathrm{THz}$ has no phase singularity, because this line has no dark strip (compare this image to the Fig. 6 'FC1' first row, which shows that Gaussian SH focuses in a line without dark strip). In the case of vortex $\mathrm{THz}$ singular phase of the beam (more precisely, destructive interference between parts of opposite phase) is responsible for the appearance of the dark strip in the line of focused singular $\mathrm{THz}$ beam, which is clearly seen in the Fig. 7 'FC2' second row (compare this image to the 


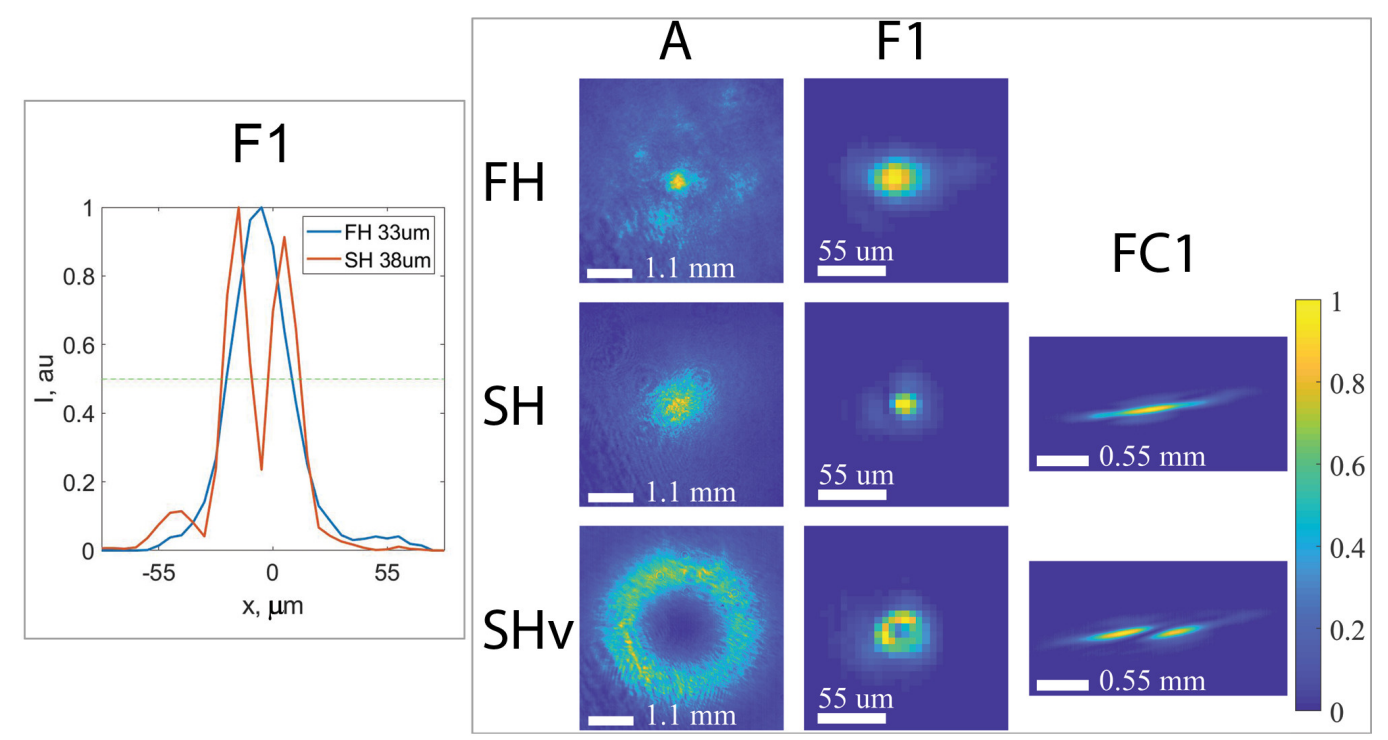

Figure 6. Panel on the right: Images of fundamental (FH) and second harmonic for Gaussian (SH) and vortex (SHv) states. Column labeling correspond to locations where images were acquired as specified in Fig. 1. A is for location before focusing lens, F1 - in the focus of this lens, FC1 - in focus of cylindrical mirror. See text for details. Colorbar on the right shows normalized intensity for all subimages. Panel on the left: Spatial overlap of Gaussian FH and vortex SH at the focus of main focusing lens at position F1. Indicated beam waists are at the FWHM level.

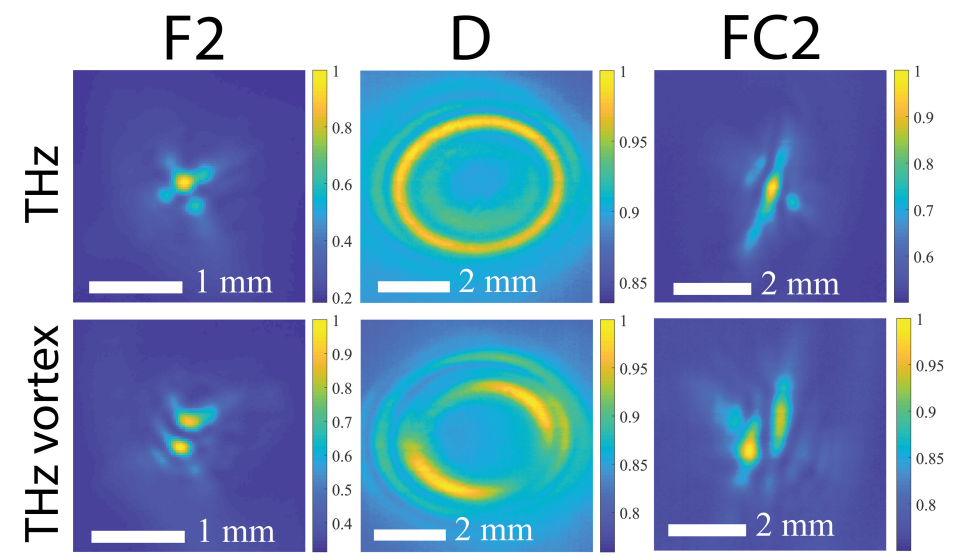

Figure 7. Experimental images of the $\mathrm{THz}$ beam generated from Gaussian (first row) and vortex SH (second row) pump beams. Column labeling correspond to locations where images were acquired as specified in Fig. 1. F2 correspond to the focus of parabolic mirror, D - collimated THz, FC2 - focus of cylindrical mirror. The FH beam was Gaussian in both cases. See text for details.

Fig. 6 'FC1' second row, which shows that vortex SH focuses in a line with dark strip). One dark strip suggests that similarly to the vortex $\mathrm{SH}$, topological charge of the generated vortex $\mathrm{THz}$ is $\left|l_{T H z}\right|=1$. Used method of topological charge determination based on astigmatic transformation of singular beams by cylindrical mirror ${ }^{28}$ is still valid even in the presence of intensity modulation of the generated THz.

Fig. 8 shows intensity distribution of the generated vortex $\mathrm{THz}$ beam at different delays of $\mathrm{FH}$ pulse with respect to the SH pulse. First row indicates time delay in femtoseconds. Second shows intensity distribution of the generated $\mathrm{THz}$ radiation. Third row shows the lines drown across two minima points in the intensity modulated ring of intensity. Over delay of $240 \mathrm{fs}$ (or light travelling distance of $72 \mu \mathrm{m}$ ) the intensity of $\mathrm{THz}$ radiation makes a turn of about 60 degrees. The change in orientation in this case is attributed to the propagation effects of vortex $\mathrm{THz}$ beam and to the shift of the central frequency with the change of delay as shown in the rightmost column in Fig. 8. Note, that in this case the shown spectra are obtained in the case of non-vortex 


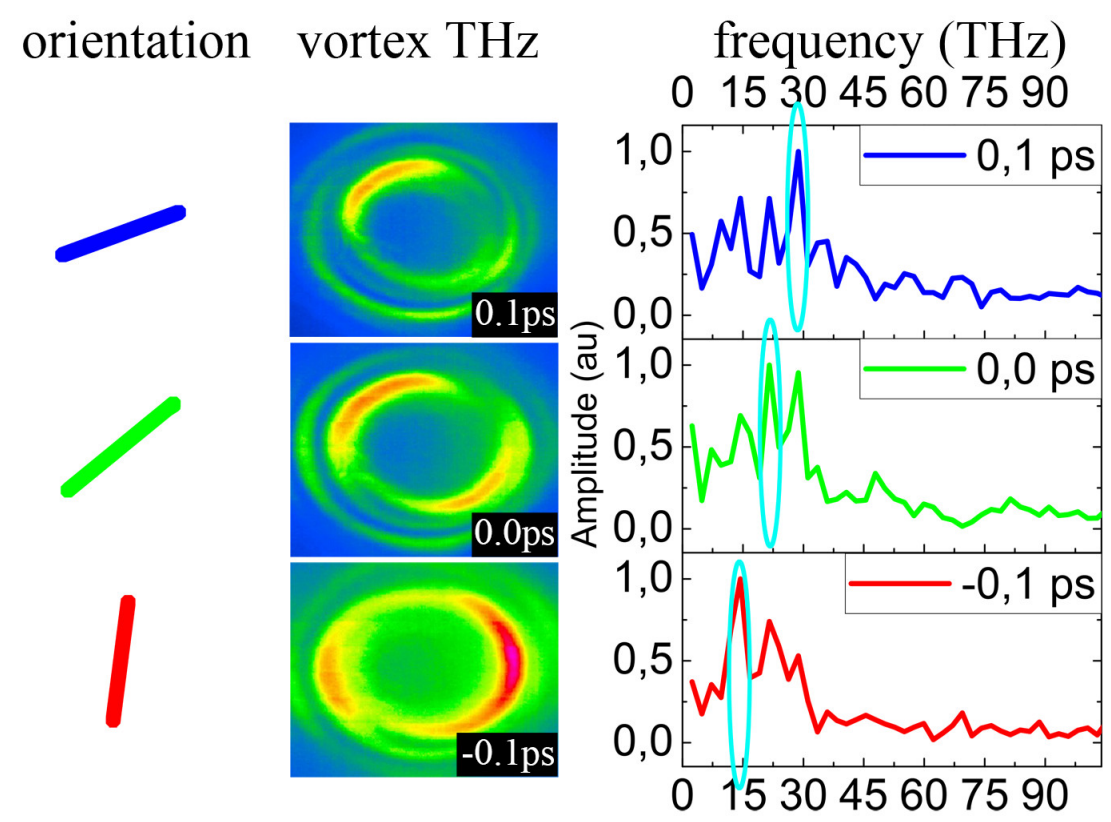

Figure 8. Orientation (schematically shown in the leftmost column) of vortex $\mathrm{THz}$ intensity distribution (middle column) versus time delay between $\mathrm{FH}$ and $\mathrm{SH}$ (indicated in the labels). Rightmost column shows spectrum at specified time delay for non-vortex $\mathrm{THz}$ (taken from Fig. 3), which picks at around 15, 20, and $30 \mathrm{THz}$ for $-0.1,0,0.1$ ps time delay, correspondingly. See text for details.

$\mathrm{THz}$ (Fig. 3) and shown here only for the reference to highlight the change of the central wavelength with the change of time delay between $\mathrm{FH}$ and $\mathrm{SH}$ pulses.

Spectra of generated $\mathrm{THz}$ with and without phase singularity is shown in the bottom-right panel of Fig. 9. In the case of vortex $\mathrm{THz}$ maximum spectral intensity is around $30 \mathrm{THz}$, while $\mathrm{THz}$ without phase singularity has maximum around $20 \mathrm{THz}$. This may be attributed to lower ionization rate at the periphery of ionizing $\mathrm{FH}$ where SH has maximum intensity and due to transverse phase variation.

Although astigmatic transformation of generated vortex $\mathrm{THz}$ beam by cylindrical mirror was used as a primary detection method of phase singularity. Additionally, we can look at the intensity variation of the generated $\mathrm{THz}$ with change of the relative phase between $\mathrm{FH}$ and $\mathrm{SH}$. The maximum $\mathrm{THz}$ yield is expected for a relative phase $(\phi+\theta)=\pi / 2$ and $3 \pi / 2$ between FH and SH. Since the phase of the SH vortex beam changes by $2 \pi$ over the full azimuthal angle, while the phase of the $\mathrm{FH}$ is constant along this angle, the intensity of the $\mathrm{THz}$ vortex beam has two minima and maxima. Changing the relative phase between FH and SH should result in position change (rotation) of these maxima and minima. To verify this property, the $\mathrm{THz}$ vortex intensity distribution was filmed by the thermal camera. The relative phase between FH and SH was not controlled but was fluctuating due to long beam pass distances ( $>5$ meters for each harmonic), vibrations in the room/building, temperature variations, etc. Because of the random nature of the fluctuations, the $\mathrm{THz}$ vortex intensity distribution jitters and makes a full rotation once in a while. The typical time constant for these phase variations was of the order of $5 \mathrm{~s}$, therefore we were able to register both the relatively stable $\mathrm{THz}$ patterns or the randomly rotating ones. The thermal camera takes 50 frames per second, corresponding to 20 milliseconds delay between subsequent frames. 12 consecutive frames (duration $240 \mathrm{~ms}$ ) were chosen from a $1 \mathrm{sec}$ long video to demonstrate the rotation of the intensity distribution due to fluctuations of the relative phase as shown in top of Figure 9. Locations of the maxima and minima were found and plotted by a home written matlab script. The top-rightmost part of the Fig. 9 shows that minima of the beam change their locations in the beam by 180 degrees during the full video sequence. The colorbar on the right indicates the number of the frame; changing from cyan for the first frame to margenta for the twelfth frame. Locations of the maxima, color-coded to change from dark to light brown, demonstrate a similar behaviour. A video sequence of the intensity distribution rotation is available via 


\section{THz vortex}

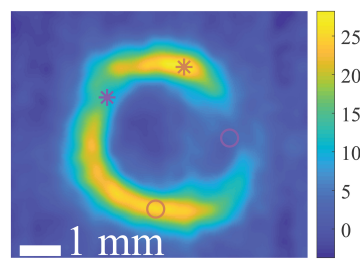

$\mathrm{THz}$

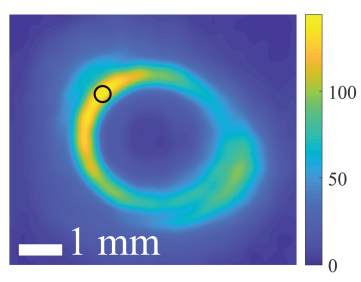

\section{Locations of maxima Locations of minima}

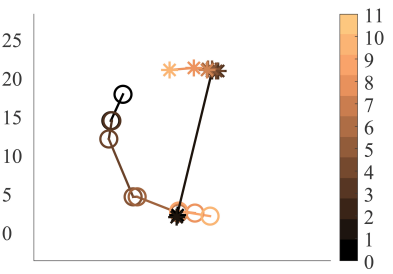

Locations of maxima

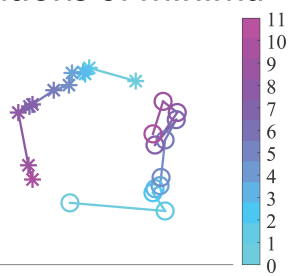

THz spectrum

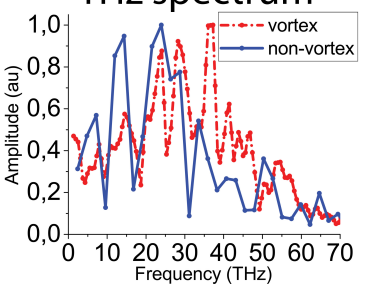

Figure 9. Left: Intensity distribution of vortex THz beam (top, video 1: http://dx.doi.org/doi.number.goes.here) and THz without phase singularity (bottom, video 2: http://dx.doi.org/doi.number.goes.here) versus fluctuations of relative phase $\phi$ between FH and vortex or Gaussian SH. Shown is one frame from a 12-frame long video-sequence. Positions of maxima are shown by brown circle and star; positions of minima are shown by magenta circle and star. Colorbar indicates intensity in a.u. Center: Locations of maxima obtained from the video sequence on the left are shown. Colorbar indicates frame number. Right-top: Locations of minima obtained from the video sequence on the left are shown. Colorbar indicates frame number. Right-bottom: Typical spectral amplitude of the generated $\mathrm{THz}$ in the case of vortex SH (red dash-dot "vortex") and Gaussian SH (blue solid line "non-vortex").

the link: video 1: http://dx.doi.org/doi.number.goes.here. On contrary, the intensity distribution of the $\mathrm{THz}$ beam without phase singularity does not change (Figure 9 bottom). The fact that the THz beam has a distinct maximum is due to slight misalignment of transverse locations of the FH and $\mathrm{SH}$ beams. The maximum of the $\mathrm{THz}$ beam slightly oscillates around the same position due to (flaws in) pointing stability of the laser. The video sequence available via the link (video 2 http://dx.doi.org/doi.number.goes.here) demonstrates a breathing (inout) character of the intensity of the $\mathrm{THz}$ beam. While filming $\mathrm{THz}$ radiation with and without phase singularity alignment of the setup was not changed. Yet, the generated $\mathrm{THz}$ beam, in the cases of vortex and Gaussian $\mathrm{SH}$ pump, shows qualitatively different behaviour consistent with the theoretical predictions.

\section{CONCLUSIONS}

We have investigated the properties of $\mathrm{THz}$ radiation generated in air plasma by a focused bichromatic femtosecond laser pulses with independent control of properties of fundamental and second harmonic beams. We demonstrated that the spatial (both transverse and longitudinal) misalignment of harmonics does not significantly shift spectral distribution of the generated $\mathrm{THz}$ pulse. The same is true for the variation of energy of fundamental harmonic. On the contrary, the spectrum of the generated $\mathrm{THz}$ beam shifts towards higher frequencies with fundamental harmonic arriving prior to second harmonic. Further, we have investigated the properties of the $\mathrm{THz}$ radiation generated in air plasma when one of second harmonic was an optical vortex. The presence of a phase singularity in the generated $\mathrm{THz}$ beam in this case was confirmed by astigmatic transformation of the singular $\mathrm{THz}$ beams in the focus of a cylindrical lens. However, in contrast to other nonlinear processes (second harmonic generation, parametric generation, etc.) the $\mathrm{THz}$ radiation generated by electron currents in a plasma filament can not be characterized as a THz vortex beam in the 'classical' sense, such as a Laguerre-Gaussian beam. Instead, the intensity of the $\mathrm{THz}$ beam is modulated along the beam azimuthal angle and contains two minima between two lobes of maximum intensity. This is because the relative phase between two harmonics spatially varies when one of the pumps is a vortex, affecting the spatial efficiency of $\mathrm{THz}$ generation. The change of this intensity modulation with relative phase between optical harmonics can additionally be used to show qualitative differences of the vortex $\mathrm{THz}$ and $\mathrm{THz}$ without phase singularity. One of the benefits of the 
demonstrated scheme is the generation of ultra-broadband vortex $(>40 \mathrm{THz})$ in contrast to the spectrally limited external $\mathrm{THz}$ shaping. We believe that the presented investigations will open new routes towards an active control of ultra-broadband $\mathrm{THz}$ beam properties. For example, different combinations of topological charges of fundamental and second harmonic pulses give great flexibility for the generation of exotic singular $\mathrm{THz}$ pulses.

\section{ACKNOWLEDGMENTS}

This project has received funding from the European Union's Horizon 2020 research and innovation programme under grant agreement no 654148 Laserlab-Europe. This research was funded by a grant (No. S-MIP-19-46) from the Research Council of Lithuania. MI, DB, and VV are grateful to Titas Gertus from Workshop of Photonics, Lithuania, for the S-waveplate used in this experiment as an optical vortex generator.

\section{REFERENCES}

[1] Ivanov, M., Thiele, I., Bergé, L., Skupin, S., Buožius, D., and Vaičaitis, V., "Intensity modulated terahertz vortex wave generation in air plasma by two-color femtosecond laser pulses," accepted to Opt. Lett. (2019).

[2] Ivanov, M., Thiele, I., Bergé, L., Skupin, S., Buožius, D., and Vaičaitis, V., "Terahertz vortex wave generation in air plasma by two-color femtosecond laser pulses," arXiv e-prints, arXiv:1906.06243 (Jun 2019).

[3] Jepsen, P. U., Cooke, D. G., and Koch, M., "Terahertz spectroscopy and imaging modern techniques and applications," Laser Photonics Rev 5 (1), 124-166 (2011).

[4] Kealhofer, C., Schneider, W., Ehberger, D., Ryabov, A., Krausz, F., and Baum, P., "All-optical control and metrology of electron pulses," Science 352, 429-433 (2016).

[5] Cook, D. J. and Hochstrasser, R. M., "Intense terahertz pulses by four-wave rectification in air," Opt. Lett. 25(16), 1210-1212 (2000).

[6] B. Clough, J. D. and Zhang, X.-C., "Laser air photonics: beyond the terahertz gap," Mater. Today 15(1-2), 50-58 (2012).

[7] Clerici, M., Peccianti, M., Schmidt, B. E., Caspani, L., Shalaby, M., Giguere, M., Lotti, A., Couairon, A., Legare, F., Ozaki, T., Faccio, D., and Morandotti, R., "Wavelength scaling of terahertz generation by gas ionization," Phys. Rev. Lett. 110, 253901 (2013).

[8] de Alaiza Martinez, P. G., Babushkin, I., Berge, L., Skupin, S., Cabrera-Granado, E., Kohler, C., Morgner, U., Husakou, A., and Herrmann, J., "Boosting terahertz generation in laser-field ionized gases using a sawtooth wave shape," Phys. Rev. Lett. 114(18), 183901 (2015).

[9] Vaicaitis, V., Balachninaite, O., Morgner, U., and Babushkin, I., "Terahertz radiation generation by threecolor laser pulses in air filament," Journal of Applied Physics 125(17), 173103 (2019).

[10] Ji, W., Lee, C.-H., Chen, P., Hu, W., Ming, Y., Zhang, L., Lin, T.-H., Chigrinov, V., and Lu, Y.-Q., "Meta-q-plate for complex beam shaping," Scientific Reports 6(25528) (2016).

[11] Stanislovaitis, P., Ivanov, M., Matijosius, A., Smilgevicius, V., and Gertus, T., "Generation of radially polarized beams and higher order polarization singularities by optical parametric amplification of optical vortices," Optical Engineering 56(9), 095101 (2017).

[12] Durnin, J., "Exact solutions for nondiffracting beams. i. the scalar theory," J. Opt. Soc. Am. A 4(4), 651-654 (1987).

[13] Vaicaitis, V. and Paulikas, S., "Formation of bessel beams with continuously variable cone angle," Optical and Quantum Electronics 35(11), 1065-1071 (2003).

[14] Siviloglou, G. and Christodoulides, D., "Accelerating finite energy airy beams," Opt. Lett. 32(8), 979-981 (2007).

[15] Hell, S. W. and Wichmann, J., "Breaking the diffraction resolution limit by stimulated emission: stimulatedemission-depletion fluorescence microscopy," Opt. Lett. 19(11), 780-782 (1994).

[16] Ivanov, M. and Hanstorp, D., "Controlled spin of a nonbirefringent droplet trapped in an optical vortex beam," Optics Communications 427, 152 - 157 (2018).

[17] He, J., Wang, X., Hu, D., Ye, J., Feng, S., Kan, Q., and Zhang, Y., "Generation and evolution of the terahertz vortex beam," Opt. Express 21(17), 20230-20239 (2013). 
[18] Imai, R., Kanda, N., Higuchi, T., Konishi, K., and Kuwata-Gonokami, M., "Generation of broadband terahertz vortex beams," Opt. Lett. 39(13), 3714-3717 (2014).

[19] Miyamoto, K., Kang, B. J., Kim, W. T., Sasaki, Y., Niinomi, H., Suizu, K., Rotermund, F., and Omatsu, T., "Highly intense monocycle terahertz vortex generation by utilizing a tsurupica spiral phase plate," Scientific Reports 6, 38880 (2016).

[20] Ge, S., Chen, P., Shen, Z., Sun, W., Wang, X., Hu, W., Zhang, Y., and Lu, Y., "Terahertz vortex beam generator based on a photopatterned large birefringence liquid crystal," Opt. Express 25(11), 12349-12356 (2017).

[21] Ge, S.-J., Shen, Z.-X., Chen, P., Liang, X., Wang, X.-K., Hu, W., Zhang, Y., and Lu, Y.-Q., "Generating, separating and polarizing terahertz vortex beams via liquid crystals with gradient-rotation directors," Crystals 10(7), 314 (2017).

[22] Minasyan, A., Trovato, C., Degert, J., Freysz, E., Brasselet, E., and Abraham, E., "Geometric phase shaping of terahertz vortex beams," Opt. Lett. 42(1), 41-44 (2017).

[23] Liu, C., Liu, J., Niu, L., Wei, X., Wang, K., and Yang, Z., "Terahertz circular airy vortex beams," Scientific Reports 7, 3891 (2017).

[24] Wu, Z., Wang, X., Sun, W., Feng, S., Han, P., Ye, J., Yu, Y., and Zhang, Y., "Vectorial diffraction properties of thz vortex bessel beams," Opt. Express 26(2), 1506-1520 (2018).

[25] Lin, Q., Zheng, S., Song, Q., Zeng, X., Cai, Y., Li, Y., Chen, Z., Zha, L., Pan, X., and Xu, S., "Generation of terahertz vortex pulses without any need of manipulation in the terahertz region," Opt. Lett. 44(4), 887-890 (2019).

[26] Dhaybi, A. A., Degert, J., Brasselet, E., Abraham, E., and Freysz, E., "Generation of terahertz vortex pulses without any need of manipulation in the terahertz region," J. Opt. Soc. Am. B 36(1), 12-18 (2019).

[27] Gecevicius, M., Ivanov, M., Beresna, M., Matijosius, A., Tamuliene, V., Gertus, T., Cerkauskaite, A., Redeckas, K., Vengris, M., Smilgevicius, V., and Kazansky, P. G., "Toward the generation of broadband optical vortices: extending the spectral range of a q-plate by polarization-selective filtering," J. Opt. Soc. Am. B 35, 190-196 (2018).

[28] Denisenko, V., Shvedov, V., Desyatnikov, A. S., Neshev, D. N., Krolikowski, W., Volyar, A., Soskin, M., and Kivshar, Y. S., "Determination of topological charges of polychromatic optical vortices," Opt. Express 17, 23374-23379 (2009).

[29] Klarskov, P., Strikwerda, A. C., Iwaszczuk, K., and Jepsen, P. U., "Experimental three-dimensional beam profiling and modeling of a terahertz beam generated from a two-color air plasma," New Journal of Physics 15(7), 075012 (2013).

[30] Vaicaitis, V., Ivanov, M., Adomavicius, K., Svirskas, Z., Morgner, U., and Babushkin, I., "Influence of laser-preformed plasma on thz wave generation in air by bichromatic laser pulses," Laser Physics 28(9), 095402 (2018). 